\title{
(2) OPEN ACCESS \\ Complex interplay between moral distress and other risk factors of burnout in ICU professionals: findings from a cross-sectional survey study
}

\author{
Niek Kok (1D ,' Jelle Van Gurp, ${ }^{1}$ Johannes G van der Hoeven, ${ }^{2}$ \\ Malaika Fuchs, ${ }^{3}$ Cornelia Hoedemaekers, ${ }^{2}$ Marieke Zegers (i) ${ }^{2}$
}

- Additional supplemental material is published online only. To view, please visit the journal online (http://dx.doi. org/10.1136/bmjgs-2020012239).

${ }^{1}$ IQ Healthcare, Radboudumc, Nijmegen, The Netherlands ${ }^{2}$ Department of Intensive Care Medicine, Radboud University Medical Center, Nijmegen, The Netherlands

${ }^{3}$ Department of Intensive Care Medicine, Canisius Wilhelmina Hospital, Nijmegen, The Netherlands

\section{Correspondence to} Mr Niek Kok, IQ healthcare, Radboudumc, Nijmegen, Nijmegen, The Netherlands; Niek.Kok@radboudumc.nl

Received 21 August 2020 Accepted 16 June 2021
Check for updates

(c) Author(s) (or their employer(s)) 2021. Re-use permitted under CC BY-NC. No commercial re-use. See rights and permissions. Published by BMJ.

To cite: Kok N, Van Gurp J, van der Hoeven JG, et al. BMJ Qual Saf Epub ahead of print: [please include Day Month Year]. doi:10.1136/ bmjqs-2020-012239

\begin{abstract}
Background Burnout threatens intensive care unit (ICU) professionals' capacity to provide high-quality care. Moral distress is previously considered a root cause of burnout, but there are other risk factors of burnout such as personality, work-life balance and culture. This study aimed to disentangle the associations of ICU professionals' moral distress and other risk factors with the components of burnout-emotional exhaustion, depersonalisation and personal accomplishmentsuggesting informed burnout prevention strategies. Methods Cross-sectional survey completed in 2019 of ICU professionals in two Dutch hospitals. The survey included validated measure for burnout (the Dutch Maslach Burnout Inventory), moral distress (Moral Distress Scale), personality (short Big Five Inventory), work-home balance (Survey Work-Home Interaction Nijmegen) and organisational culture (Culture of Care Barometer). Each of the three components of burnout was analysed as a separate outcome, and for each of the components, a separate regression analysis was carried out.
\end{abstract}

Results 251 ICU professionals responded to the survey (response rate: $53.3 \%$ ). Burnout prevalence was $22.7 \%$. Findings showed that moral distress was associated with emotional exhaustion ( $\beta=0.18,95 \% \mathrm{Cl} 0.9$ to 0.26 ) and depersonalisation ( $\beta=0.19,95 \% \mathrm{Cl} 0.10$ to 0.28 ) and with increased emotional exhaustion mediated by negative work-to-home spillover $(\beta=0.09,95 \% \mathrm{Cl}$ 0.04 to 0.13$)$. Support from direct supervisors mitigates the association between moral distress and emotional exhaustion ( $\beta=0.16,95 \% \mathrm{Cl} 0.04$ to 0.27 ).

Conclusions Understanding moral distress as a root cause of burnout is too simplified. There is an important interplay between moral distress and work-home imbalance. Interventions that support individual coping with moral distress and a work-home imbalance, and the support of direct supervisors, are paramount to prevent burnout in physicians and nurses.

\section{INTRODUCTION}

Burnout syndrome (BOS) threatens the health and well-being of intensive care unit (ICU) professionals as well as the capacity to provide safe, timely, efficient and people-centred care. Consequently, there is a call for interventions that prevent BOS in ICUs. BOS is considered to have three components: emotional exhaustion, depersonalisation-a lack of empathy that adversely affects patient contact in the form of cynical and negative attitudes, and a low sense of personal accomplishment. Progressive severity in any of these components is said to match the pathology of depression. ${ }^{1-3}$ Furthermore, BOS is related to suicidal thoughts, absenteeism, decreased professionalism and decreased quality of care, substance abuse, increased staff turnover and medical errors. ${ }^{4-8}$ Especially during crises such as the COVID-19 pandemic, burnout poses a risk to ICU professionals as they are called on to be flexible and resilient while under more extreme circumstances than usual.

Moral distress is commonly presented as a root cause of burnout. ${ }^{910}$ It is defined as a psychological response to morally challenging situations such as those of moral constraint or moral conflict, or both. ${ }^{11}$ Typically, moral distress arises in situations where ICU professionals are confronted with ethical issues that lack morally satisfying responses and feel unable to act according to internally held values and perceived obligations. ${ }^{12}$ Moral distress may result from internal constraints such as personal convictions pertaining to prescriptive cultural or religious value systems, or from external constraints such as demands of patients, families or colleagues, and rules 
stipulated by protocol or law. An example is the decision to continue treating a patient when this is personally considered futile because the patient's family cannot be convinced of the treatment's futility. Consequently, the professional is unable to act according to their personal values. Several interventions have been proposed through which ICU departments may attempt to mitigate moral distress and/or its adverse consequences. ${ }^{9}{ }^{10} 12-16$ Evidence gained in randomised controlled trials as to whether these interventions reduce burnout is, however, scarce. ${ }^{16-18}$

Previous studies have shown that personality traits such as neuroticism are conducive of developing burnout, whereas agreeableness and extraversion are protective factors. ${ }^{19} 20$ An imbalance between one's work and private lives can lead to burnout, ${ }^{21}{ }^{22}$ while a constructive culture at work offers protection. ${ }^{23-25}$ However, these factors have not yet been studied alongside moral distress. It is therefore unclear how they relate to moral distress and how to account for them when devising an intervention with the aim of preventing moral distress and burnout.

Personality, work-home conflicts and culture possibly influence the association between moral distress and burnout. It has been hypothesised that neurotic individuals may more easily become burned out from experiencing moral distress. ${ }^{19}$ Compared with others, neurotic individuals are predisposed toward negative feelings and unfair treatment. Hence, those high in neuroticism can be expected to experience more difficulties in coping with the unfairness or injustice implicated in morally distressing situations. ${ }^{26}$ Workplace culture is hypothesised to have similar effects: moral distress might not lead to burnout in open, supportive cultures that provide professionals with the means to communally cope with moral distress. Furthermore, this study conjectures that moral distress plays a role in work-home conflicts. Moral distress originates in the workplace, and if it persists beyond working hours, it may hamper enjoyment of private life. Conversely, private affairs could also stimulate moral distress at work as ICU professionals may be more vulnerable to morally distressing events that reflect some of the difficult situations they encounter in their own lives.

In short, moral distress is likely to be part of a complex interplay of mediating and moderating factors that develop into burnout. Mediator variables are associated with burnout symptoms as well as being associated with each other. Moderator variables influence the sign or strength of a relationship. For instance, a certain degree of moral distress could lead to burnout in a neurotic ICU professional, whereas a similar degree of moral distress does not lead to burnout in a less neurotic professional. In order to fine-tune interventions aimed at reducing burnout, this study sets out to disentangle the associations between moral distress, personality traits, work-home balance and culture and explore potential mediators and moderators in the associations between all these risk factors and the three components of BOS-emotional exhaustion, depersonalisation and low personal accomplishment.

\section{METHODS}

\section{Design and study population}

This is a cross-sectional study using survey data collected between October 2019 and January 2020 from six Dutch ICUs in total: five ICUs based in a university medical centre (three adult units, one adult step-down unit and one paediatric ICU) plus a sixth adult ICU based in a separate teaching hospital. The survey was designed using LimeSurvey, a web-based survey tool.

\section{Outcome measures and data collection}

Burnout was measured using the validated Dutch translation of the Maslach Burnout Inventory (MBI), known as the MBI-NL for healthcare workers, sometimes referred to as the Utrecht Burnout Scale. While the original MBI consists of 22 items, a confirmatory factor analysis performed by the translators of the MBI showed that two items should be omitted from the Dutch version. ${ }^{27}$ The MBI-NL thus consists of 20 items measuring emotional exhaustion (8 items), depersonalisation (5 items) and personal accomplishment (7 items), all scored on a 7-point Likert scale ranging from never (0) to daily (6). We calculate the average score of respondents for emotional exhaustion, depersonalisation and personal accomplishment by adding up the item scores in each scale and dividing those by the total number of items that measure the subscale.

There is a distinction in the way in which average score of respondents for the subscales are calculated, and in the transformation of the item scores to a definition of BOS. Solely for the purpose of comparing our prevalence estimate of burnout with other studies and not for further statistical analysis, this paper reports prevalence according to a commonly used method, ${ }^{9}$ where a 'total score' for burnout is calculated: the scores on all items for emotional exhaustion and depersonalisation are added and the scores on the personal accomplishment items are subtracted. Respondents with cumulative scores higher than -9 are classified as having symptoms of burnout. In the estimation using this method, we correct for the two omitted items in the Dutch MBI, after which it is comparable to the original version.

Respondents were asked for their age, gender, profession, years in their current job, marital status and the age of their youngest child. Moral distress was measured using the 21-item Moral Distress Scale (MDS-R). ${ }^{28}$ Items describe morally distressing events which respondents then rate on $0-4$ scales as to how often these occur (frequency) and how distressing they are (intensity). A composite score is calculated by multiplying frequency by intensity and subsequently 
adding all the individual item scores together giving an overall score between 0 and $336 .{ }^{29}$ Personality was measured with a short version of the Big Five Inventory (BFI) using a five-point (1-5) Likert scale. ${ }^{30}$ Negative work-home and home-work spillovers were assessed using the Survey Work-Home Interaction Nijmegen with a 5-point (0-4) Likert scale. ${ }^{31} 32$ The concepts measured by this instrument are formally called work-home interactions. In this paper, we will refer to these as work-home or home-work 'spillovers'. A sample negative work-to-home spillover item is 'How often does it happen that you find it difficult to fulfil your domestic obligations because you are constantly thinking about your work?' The data were supplemented with data using the Culture of Care Barometer (CoCB) and the team climate scale of the Safety Attitudes Questionnaire. The CoCB measures four aspects of culture: organisational values, support from direct supervisors, relationships with colleagues and job autonomy. ${ }^{33}$ The team climate scale measures team climate with a 1-5 Likert scale. ${ }^{34}$ Online supplemental file A provides a detailed description of each instrument. These two instruments were included as part of the survey for the teaching hospital, but the data separately gathered for the university medical centre 3 months prior to this study.

\section{Statistical analysis}

Numerous different cut-off values for the three components have been suggested for calculating a BOS score and the choice is somewhat arbitrary. ${ }^{35-37}$ Therefore, as recommended in a previous study, multiple prevalence estimates of burnout were reported using a range of cut-off values. We report the range of BOS prevalences but carried out a separate statistical analysis for each BOS component using the average item score of respondents in emotional exhaustion, depersonalisation and personal accomplishment as described previously. ${ }^{36}$

We applied independent $t$-tests for differences in means, or where appropriate, non-parametric MannWhitney $U$ test for differences in the distribution of the data between physicians and nurses. Univariate regression analyses were used to assess the association between each individual risk factor and each BOS component, followed by multivariable regression analyses including moral distress, the demographics and all the subdomains of personality and work-home balance. In order to better compare the magnitude of associations across different scales, we calculated z-scores for each continuous scale-that is, moral distress, personality variables, work-home interactions, culture variables, age, years in current job and working hours/week. The scales transformed into z-scores were used for regression analysis.

We analysed missing values and report the degrees of missingness. Variables with a substantial amount of missing values were dropped from the analysis.
Analyses containing all variables have been added to the online supplemental file.

A regression-based mediation analysis was performed based on 10000 bootstrap samples to explore whether moral distress was part of a chain of mediating variables for each of the three burnout domains. We performed mediation analysis on two conditions: that there could be a plausible indication of a cause-effect relationship and, second, that independent and mediating variables were all significantly associated with the same burnout component in the multiple regression model. The plausibility condition implied that sociodemographic variables and personality subdomains could not be considered as mediator variables. Personality traits are considered to be relatively stable in an individual over time and across situations ${ }^{19}$; therefore, like an individual's age or gender, they cannot be said to be 'caused' by moral distress and cannot be mediating variables in models where moral distress is an independent variable. From these criteria, it appeared that eight models were eligible for mediation analysis.

A regression-based moderation analysis was performed to identify significant moderators that affect either the sign or the strength of the relationship between moral distress and BOS. We considered all variables-sociodemographics, personality subscales, work-home balance and culture subdomains-as potential moderators. This led to analysis of 19 moderation models for each of the three outcomes. Only significant moderators are reported. Because of the exploratory purpose of mediation and moderation analyses in this article, alpha inflation was not addressed. ${ }^{38}$

Given that the data in this study were hierarchical, with the professionals nested within six ICUs, fixedeffect dummies were included in all the multivariable analyses, including the mediation and moderation analyses, to account for multilevel effects. With six ICUs, a fixed effects approach is appropriate. Significance is assumed for $\mathrm{p}$ values below 0.05. Statistical analyses were performed using SPSS and the mediation and moderation assessments used Hayes' PROCESS macro. ${ }^{39}$

\section{RESULTS}

Surveys were sent to 471 ICU professionals of whom 251 replied, a response rate of $53.3 \%$. Table 1 presents sociodemographic characteristics of the respondents, of whom $73.7 \%$ were female and $21.5 \%$ worked as physicians.

Physicians scored higher on depersonalisation and had a stronger sense of personal accomplishment than nurses (table 2). There was no significant difference in levels of moral distress between physicians and nurses. Physicians less frequently experienced negative hometo-work spillovers than nurses.

Based on a commonly used method for calculating burnout symptom prevalence using the $\mathrm{MBI},{ }^{40-44}$ the 
Table 1 Participant characteristics outcome measures and risk factors of BOS comparing physicians versus nurses

\begin{tabular}{|c|c|c|c|}
\hline & Total, $\mathrm{n}=251$ & ICU physicians, $\mathrm{n}=53$ & ICU nurses, n=194† \\
\hline Variable & Mean (SD) or percentage & Mean (SD) or percentage & Mean (SD) or percentage \\
\hline \multicolumn{4}{|l|}{ Sex $(\%)$} \\
\hline Female & 73.7 & 49.1 & 80.9 \\
\hline Male & 26.3 & 50.9 & 19.1 \\
\hline Age (years) (SD) & $42.5(11.4)$ & $39.7(10.9)$ & $43.3(11.6)$ \\
\hline Years in current job (SD) & $12.7(10.3)$ & $6.9(7.9)$ & $14.3(10.4)$ \\
\hline Working hours/week (SD) & $31.3(7.3)$ & $39.9(6.9)$ & $29.1(5.3)$ \\
\hline \multicolumn{4}{|l|}{ Preschool or young child at home (\%) } \\
\hline Yes & 82.9 & 81.1 & 83.0 \\
\hline No & 17.1 & 18.9 & 17.0 \\
\hline \multicolumn{4}{|l|}{ Partner (\%) } \\
\hline Yes & 82.3 & 74.0 & 84.8 \\
\hline No & 17.7 & 26.0 & 15.2 \\
\hline \multicolumn{4}{|l|}{ BOS } \\
\hline Emotional exhaustion & $1.23(0.82)$ & $1.19(0.78)$ & $1.25(0.83)$ \\
\hline Depersonalisation & $0.85(0.71)$ & $1.11 * *(0.78) \ddagger$ & $0.79 * *(0.68) \ddagger$ \\
\hline Personal accomplishment & $4.35(0.79)$ & $4.60 * *(0.62) \ddagger$ & $4.27 * *(0.82) \ddagger$ \\
\hline Moral distress & $30.70(20.20)$ & $27.69(16.40)$ & $31.85(21.10)$ \\
\hline \multicolumn{4}{|l|}{ Personality } \\
\hline Agreeableness & $4.07(0.49)$ & $4.05(0.49)$ & $4.08(0.49)$ \\
\hline Conscientiousness & $4.10(0.46)$ & $4.12(0.35)$ & $4.09(0.49)$ \\
\hline Extraversion & $3.75(0.55)$ & $3.68(0.67)$ & $3.77(0.52)$ \\
\hline Neuroticism & $2.45(0.61)$ & $2.28(0.56)$ & $2.51(0.62)$ \\
\hline Openness & $3.25(0.62)$ & $3.31(0.65)$ & $3.24(0.61)$ \\
\hline \multicolumn{4}{|l|}{ Work-home balance } \\
\hline Negative work-to-home spillover & $0.61(0.47)$ & $0.65(0.46)$ & $0.60(0.47)$ \\
\hline Negative home-to-work spillover & $0.23(0.33)$ & $0.13 *(0.24) \ddagger$ & $0.25 *(0.34) \ddagger$ \\
\hline \multicolumn{4}{|l|}{ Culture of care } \\
\hline Organisational values & $3.22(0.74)$ & $3.54(0.67)$ & $3.19(0.73)$ \\
\hline Support from supervisor & $3.64(0.61)$ & $3.78(0.66)$ & $3.63(0.59)$ \\
\hline Relationships with colleagues & $3.87(0.62)$ & $3.93(0.63)$ & $3.89(0.60)$ \\
\hline Absence of job constraints & $3.71(0.66)$ & $3.75(0.59)$ & $3.73(0.67)$ \\
\hline Teamwork climate & $3.64(0.56)$ & $3.76(0.24)$ & $3.63(0.34)$ \\
\hline
\end{tabular}

Significant results are shown in bold.

${ }^{*} \mathrm{P}<0.05,{ }^{*} \mathrm{P}<0.01$.

tData for profession was missing of four respondents.

‡Non-parametric Mann-Whitney U tests.

BOS, burnout syndrome; ICU, intensive care unit.

estimated prevalence in this study was $22.7 \%$. Online supplemental file B describes what the prevalence estimate would be if we adopted different methods, comparing our estimate with those of 25 other studies. For those $22.7 \%$ respondents with burnout symptoms, mean values for the components were $2.23(\mathrm{SD}=0.86)$ for emotional exhaustion, $1.52(\mathrm{SD}=0.79)$ for depersonalisation and $3.60(\mathrm{SD}=0.79)$ for personal accomplishment (see online supplemental file C).

\section{Missing data analysis}

For 251 respondents, we had complete data for emotional exhaustion, depersonalisation and personal accomplishment. Missing data analysis showed that $8.0 \%$ of these 251 respondents had missings for moral distress. Most other variables had very few missing variables, except for the variable whether respondents had a partner or not (5.2\%) and up to $30 \%$ for the variables measuring culture (for the exact percentages of missing values, see online supplemental file D). Given this proportion of missing datapoints for culture, these variables were dropped from the regression analysis. Results of the multivariable models that include culture are reported separately in online supplemental file E. Moreover, as it was not a variable of interest and the amount of missing values on being partnered was relatively high, this variable was dropped from the multivariable analysis after the univariable analysis indicated that it was not essential. This left 205 full cases for the multivariable analysis. 


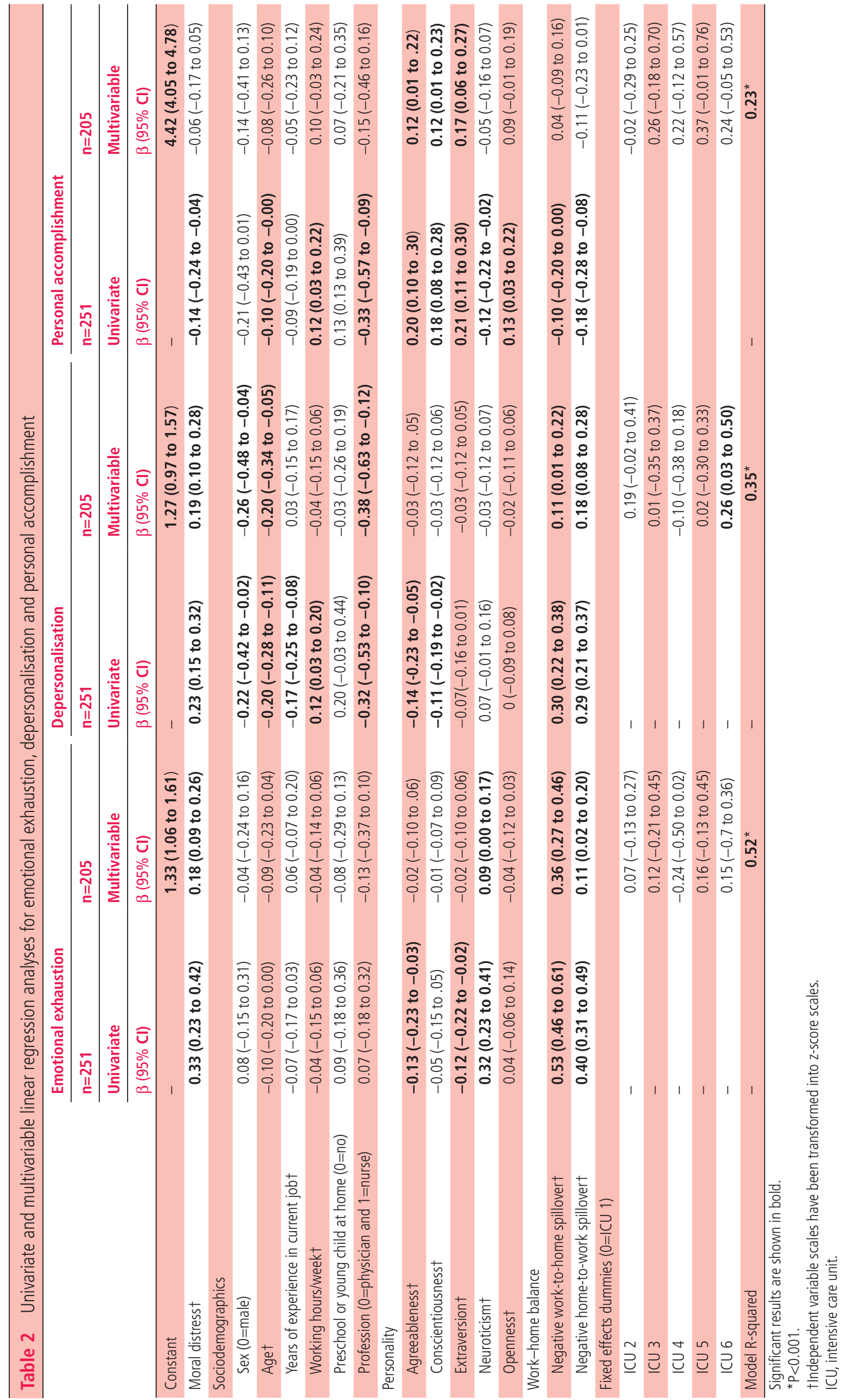




\section{Associations between risk factors and BOS \\ components}

Moral distress was positively associated for both emotional exhaustion and depersonalisation, as seen in the 'univariate' column in table 2 , and in the multivariable model. Moral distress was not associated with personal accomplishment once other variables were added to the model. Men and physicians appear particularly prone to feelings of depersonalisation, while older people were less likely to feel depersonalised. Neuroticism was associated with emotional exhaustion. The agreeableness, conscientiousness and extraversion personality traits appear to incite a heightened sense of accomplishment. Frequent spillovers of negative experiences from work to private life and vice versa were associated with greater emotional exhaustion and depersonalisation. These associations had reasonably large coefficients indicating a significant influence. For example, an increase of one $\mathrm{z}$-score on the work-to-home spillover scale was associated with a 0.36 increase in the emotional exhaustion scale. In contrast, a $\mathrm{z}$-score increase on the moral distress scale was associated with a 0.18 increase on this scale. This suggests that ICU professionals who are experiencing some negative work-to-home spillovers can more quickly slide into an unhealthy emotionally exhausted state. The regression analysis showed that higher scores on emotional exhaustion can only be reached through a combination of contributing risk factors. For examples, see box 1.

\section{Mediating and moderating factors}

Moral distress was directly associated with emotional exhaustion $(\beta=0.19, \mathrm{p}<0.05)$. However, the association between moral distress and emotional exhaustion was also mediated through negative work-to-home spillovers $(\beta=0.09)$. This indirect association was statistically significant. The sequence is depicted in figure 1 .

The moderation analysis depicted in figure 2 shows that support from supervisors moderates the association between moral distress and emotional exhaustion $(\beta=0.16, p<0.01)$. The same level of moral distress was associated with less emotional exhaustion in ICU professionals who felt supported than in those who felt less supported by the management.

\section{DISCUSSION}

This study shows that moral distress is consistently related to emotional exhaustion and depersonalisation. As demonstrated, it accounted for a relatively smaller change in emotional exhaustion compared with workhome spillovers. The magnitude of the association between moral distress and depersonalisation was, however, similar to the association between home-towork spillovers and depersonalisation. Neuroticism was consistently associated with emotional exhaustion, while agreeableness and extraversion were associated
Box 1 Examples of associations between burnout, moral distress and other risk factors of burnout

The regression model in table 2 has this form, where $Y$ can be any of the three burnout components; $\beta_{0}$ is the constant; $\beta_{1}$ is the coefficient for moral distress and $X_{1}$ is its value. Each subsequent $\beta$ stands for each other variable in the model, denoted by $i$. Lastly, the $\varepsilon$ is the error term.

$$
\mathrm{Y}=\beta_{0}+\beta_{1} \mathrm{X}_{1}+\beta_{\mathrm{i}} \mathrm{X}_{\mathrm{i}}+\epsilon
$$

The constant in each of the burnout component scales corresponds to the value of a respondent that is a male physician who works on ICU 1, without care duties for a young child, and that scores a mean value on every continuous variable. This respondent scores a 1.33 on the emotional exhaustion subscale that ranges from 0 to 6 . Previously, we have demonstrated that the $22.7 \%$ respondents with burnout symptoms on average score a 2.23 on emotional exhaustion, with an SD of 0.86 . The male physician from our example would thus need a 0.90 increase in his score to reach the mean level of emotional exhaustion within the subgroup of respondents with burnout symptoms.

This will most likely only be reached by a combination of high scores on moral distress, neurotic personality, work-to-home spillovers and home-to-work spillovers. If this physician would, for instance, score 1 z-score on moral distress and both work-to-home spillovers and home-to-work spillovers, this would be associated with a score on emotional exhaustion of 1.98-which comes close to the mean value for respondents with burnout but is still below average for this group.

The same male physician would have a troubling score on depersonalisation if he scored 1 z-score on all three risk factors: moral distress, work-to-home and home-to-work spillovers. Then, the associated value for depersonalisation would be 1.75, well above the average score of 1.52 within the subgroup of respondents with burnout symptoms.

The physician's value on personal accomplishment would be 4.42 , while the subgroup of respondents with burnout symptoms score, on average, 3.60 on personal accomplishment. The magnitude of the associations between risk factors of burnout and personal accomplishment is much smaller and most associations are not significant. Without taking statistical significance into account, we see that being a female nurse is, for instance, associated with lower levels of personal accomplishment. A personality type high in agreeableness, conscientiousness and extraversion is conducive of personal accomplishment.

with a sense of greater personal accomplishment. Levels of moral distress were similar in physicians and ICU nurses. In terms of BOS, physicians scored more highly on depersonalisation than nurses. We also found 


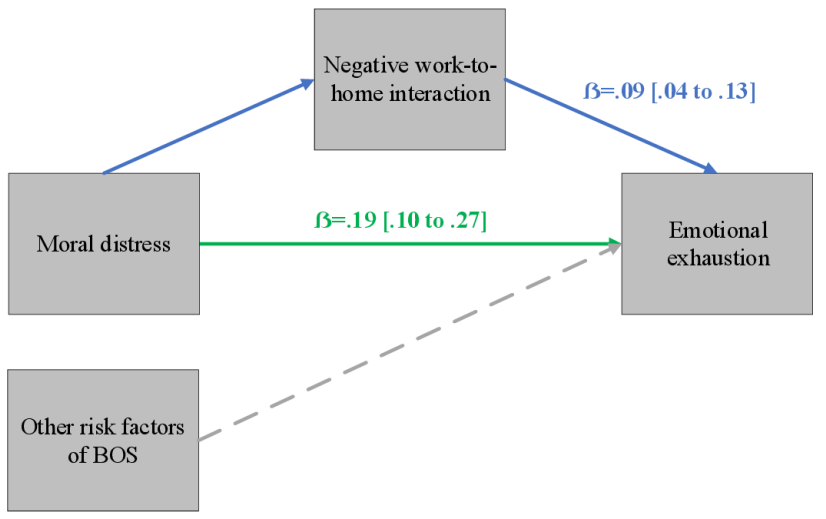

Figure 1 Associations between moral distress and emotional exhaustion through negative work-home interactions. Using Hayes PROCESS Macro, all pathways could be tested. The mediation analysis was controlled for by other risk factors of BOS, including the demographics and all the subdomains of personality reported in tables 1 and 2. BOS, burnout syndrome.

that supervisors make a difference: their support mitigates the adverse association between moral distress and emotional exhaustion.

Moral distress has been called a one-sided root cause of burnout. ${ }^{910}$ The findings from the mediational analysis show that the dynamic by which moral distress is associated with burnout is more complex. Moral distress is part of a complex chain of associations
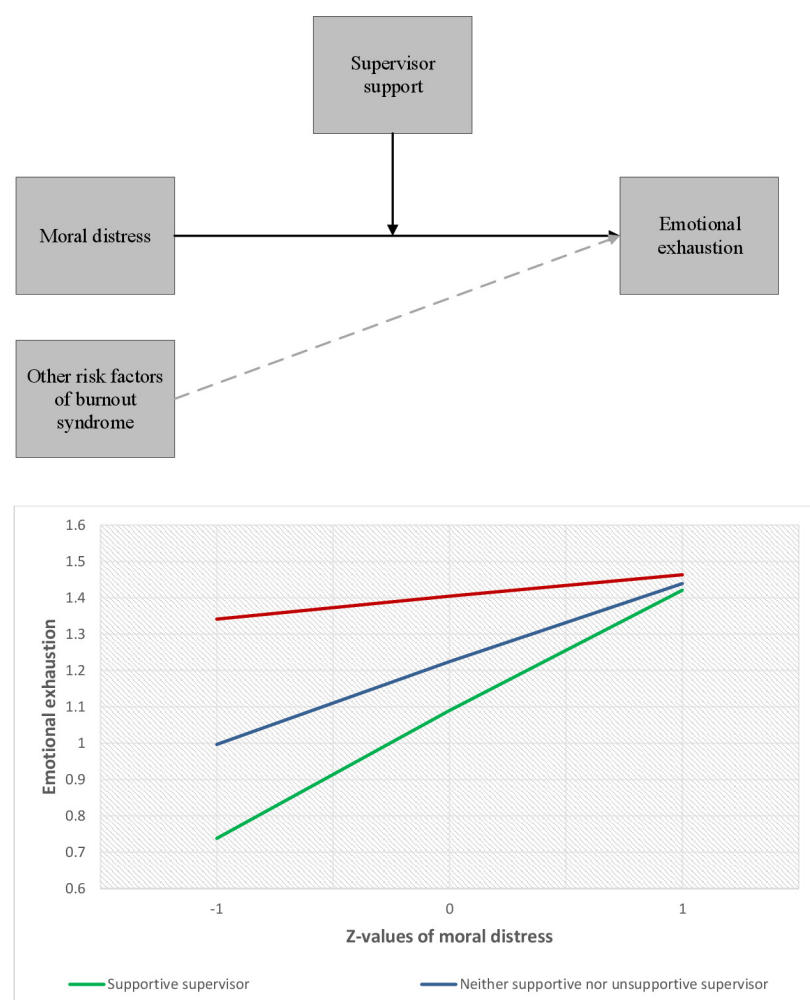

- Unsupportive supervisor

Figure 2 Association between moral distress and emotional exhaustion moderated by felt support from direct supervisors. 1The Y-axis reports emotional exhaustion on the original 7-point Likert scale ranging from never (0) to daily (6). between ICU professionals' private life and undesired outcomes such as burnout. Previous studies have revealed that negative work-home spillovers are associated with a diminishing safety culture and may have detrimental effects on health and well-being by increasing psychosomatic symptoms and physical health conditions. ${ }^{2131}$ This study suggests that some of these work-to-home spillovers have a moral character, that is, that morally distressing ICU events carry over to one's private life. The findings support a plausible chain of events which can be characterised as an onrush of psychological decompensation where some ICU professionals are simply unable to leave their work-related moral distress behind when they return home: tragic events and patient deaths continue to linger in the minds of at least some of them, potentially leading to a state of emotional exhaustion and a deteriorating capacity for coping.

It is essential to note that the directionality of the effects depicted in figure 1 cannot be drawn from a cross-sectional study. Hence, while the process we describe is plausible, we postulate it as a hypothesis which needs testing in future studies, preferably of a longitudinal kind. While other studies purport that moral distress can result in burnout, ${ }^{9} 101214$ this does not necessarily have to be the direction of the relationship. Moreover, it is not unlikely that individuals first burnout and consequently experience moral distress due to self-perceived malfunctioning. Because of having a professional identity, professionals will likely perceive of themselves as committed and connected employees. Burnout could erode these core professional virtues or values, thereby causing moral distress.

Another new finding is that support from direct supervisors can be beneficial: it helps to prevent the adverse consequences of moral distress on emotional exhaustion. In our sample, these direct supervisors were intensivists occupying management positions who oversaw junior physicians, and lead nurses who oversaw other nurses. Previous studies have shown that this support can boost the meaningfulness of work $\mathrm{w}^{45} 46$ and that professionals who worked under supervisors that were attuned to emotional signals reported less emotional exhaustion during a hospital restructuring. ${ }^{47}$ Supportive supervisors were thus found to carry other professionals through difficult times. Our findings suggest the generalisability of this moderating effect to include morally distressing events.

The findings lend support to previous findings that personality traits can increase proneness to burnout. ${ }^{19}$ Personality traits were most strongly associated with a sense of personal accomplishment, which is, however, not always seen as a key component of BOS. ${ }^{48}$

\section{Strengths and limitations}

A strength of this study is the use of only validated scales in the questionnaires to estimate outcome measures and then subjecting these measures to 
multivariable and mediation analysis. Both the MDS-R and the MBI are healthcare-oriented assessment tools. Previous validation of these instruments strengthens the evidence that there is a relation between workplace stressors and home-based stressors.

A bias which plagues BOS studies in general is that such studies tend to include an over-representation of healthy workers, a bias known as the "healthy worker effect' (HWE) ${ }^{49}$ In this study, there may be an HWE because individuals with BOS may have excluded themselves. Although we sent surveys to all the ICU professionals, including those who were absent from work for reasons of sickness, it is still possible that, due to the HWE, levels of BOS were underestimated.

Compared with similar studies with Danish, German and Belgian samples that had response rates ranging from $26 \%$ to $51 \%,{ }^{19}{ }^{50-54}$ this study had a relatively high response rate of $53.3 \%$. Additionally, for webbased surveys of health professionals, rates of under $20 \%$ are not uncommon. ${ }^{55}$ However, though better than several similar studies, this paper's response rate is still modest, and the representativeness might be limited. Some, notably Swiss studies have had very high response rates, sometimes above $80 \%$. Our sample nevertheless seems sufficiently large, and the inclusion of six ICUs across two hospitals provides a more complete picture of the situation in different types of ICUs.

The missing datapoints for culture, as well as the fact that the data on culture was collected 3 months prior to the collecting the other data, placed limitations on including culture in the models. Although not typical, cultures can shift across 3 months. Further, our moderation analysis does not allow one to conclude whether professionals with high levels of moral distress or emotional exhaustion generally received support from supervisors more frequently and more intensively. This was because we only measured the degree to which professionals felt their supervisors supported them, not how intensively they were supported or needed support. There may thus be some unmeasured variability.

Moreover, the correlational nature of the data precludes definitive cause-and-effect claims. However, cross-sectional data are not seen as an obstacle to performing a mediation analysis, ${ }^{39}$ and our mediation model is plausible, although we cannot claim causality. A longitudinal intervention study would be required to establish whether interventions aimed at reducing moral distress do indeed reduce emotional exhaustion and depersonalisation, and qualitative research is needed to explore the causal mechanisms implied in such interventions.

\section{Implications for practice}

Several interventions could help prevent BOS by addressing moral distress. For instance, moral case deliberation, in which professionals jointly reflect on ethical issues pertaining to everyday practice, has realistic prospects of helping professionals come to an understanding on morally distressing events. Although moral case deliberation is unlikely to 'solve' moral issues-as these tend not to be solved easily-it can help in making sense of one's own or another's moral burden and coming up with well-considered judgements. ${ }^{56}$

One intervention that could address negative workhome balances is to create awareness that ICU professionals all have their own things going on in their private lives. While it is impossible to ensure 'fitness to fly' for every professional at the start of their shift, awareness by professionals, and especially supervisors, of each other's private difficulties may be a step in the right direction. Second, reflection on morally distressing events at the end of a shift, a form of cooling down period, may lessen the intensity of negative moral experiences spilling over into private life. ${ }^{16}$ If such debriefings are organised towards the end of shifts, these could be meetings where professionals not necessarily resolve but make some sense of morally distressing experiences before returning home. This may remove some of the initially felt perplexity or outrage, meaning that once professionals return home, even if a moral issue has not been resolved, they will have already started processing these issues in a somewhat analytical and emotionally balanced way. There can also be downsides, however. If teams discuss morally laden events, this may trigger individuals who either did not know about the event or further outrage those that were involved. Some recent studies have studied individual-directed and organisation-directed interventions, ${ }^{17} 18$ similar efforts should be made towards research on the interventions proposed by this study, prior to widespread implementation.

The finding that support from supervisors complexly plays into the association between moral distress and emotional exhaustion leads to the very practical recommendation that ICU supervisors should stay attuned to moral experiences, give their staff room to share their concerns and the opportunity to exert some influence over moral decisions, and finally, to consistently reprimand unacceptable behaviour of professionals towards each other. One way in which supervisors could help mitigate the adverse effects of moral distress is by enabling and actively joining in the deliberations and showing a willingness to listen and to participate on an equal footing. This recommendation applies to all direct supervisors, both leading physicians and lead nurses alike.

\section{CONCLUSION}

Understanding moral distress as a unilateral root cause of burnout is too simplified. To prevent burnout and maintain high quality of care, it is essential to intervene and support individuals' understanding of moral distress and a disrupted work-home balance, and for 
ICU leaders to behave in a supportive manner towards physicians and nurses.

Contributors All authors contributed to the study conception and design. Acquisition was performed by NK, JVG, MF, $\mathrm{CH}$ and MZ. Analysis and interpretation of the data were performed by NK, JVG, HH, CH and MZ. The first draft of the manuscript was written by NK and all authors commented on previous versions of the manuscript. All authors read and approved the final manuscript.

Funding The research was supported by ZonMw, Project number 516012513.

Competing interests None declared.

Patient consent for publication Not required.

Ethics approval This study complied with ethical standards. All ICU professionals were sent an email with a link to the survey and had to provide written informed consent. The study has been approved by the regional ethics committee (CMO region Arnhem-Nijmegen: 2018-4346).

Provenance and peer review Not commissioned; externally peer reviewed.

Data availability statement Data are available upon reasonable request. All data relevant to the study are included in the article or uploaded as supplementary information. The data consist of deidentified participant data. The data are available from Niek Kok (ORCID-ID: 0000-0002-60664496).

Supplemental material This content has been supplied by the author(s). It has not been vetted by BMJ Publishing Group Limited (BMJ) and may not have been peer-reviewed. Any opinions or recommendations discussed are solely those of the author(s) and are not endorsed by BMJ. BMJ disclaims all liability and responsibility arising from any reliance placed on the content. Where the content includes any translated material, BMJ does not warrant the accuracy and reliability of the translations (including but not limited to local regulations, clinical guidelines, terminology, drug names and drug dosages), and is not responsible for any error and/or omissions arising from translation and adaptation or otherwise.

Open access This is an open access article distributed in accordance with the Creative Commons Attribution Non Commercial (CC BY-NC 4.0) license, which permits others to distribute, remix, adapt, build upon this work noncommercially, and license their derivative works on different terms, provided the original work is properly cited, appropriate credit is given, any changes made indicated, and the use is noncommercial. See: http://creativecommons.org/licenses/by-nc/4. $0 /$.

\section{ORCID iDs}

Niek Kok http://orcid.org/0000-0002-6066-4496

Marieke Zegers http://orcid.org/0000-0002-7472-6184

\section{REFERENCES}

1 Bianchi R, Boffy C, Hingray C, et al. Comparative symptomatology of burnout and depression. J Health Psychol 2013;18:782-7.

2 Wurm W, Vogel K, Holl A, et al. Depression-Burnout overlap in physicians. PLoS One 2016;11:e0149913.

3 van Dam A. Subgroup analysis in burnout: relations between fatigue, anxiety, and depression. Front Psychol 2016;7:1-9.

4 Moss M, Good VS, Gozal D, et al. An official critical care societies collaborative Statement-Burnout syndrome in critical care health-care professionals: a call for action. Chest 2016;150:17-26.

5 Mion G, Libert N, Journois D. The prevalence of burnout. Intensive Care Med 2018;44:1192-4.
6 Mion G, Boiguile K, Bidou A, et al. Burnout among French anaesthetists and intensivists: adequate progress is still lacking. Anaesth Crit Care Pain Med 2017;36:247-8.

7 Hyman SA, Shotwell MS, Michaels DR, et al. A survey evaluating burnout, health status, depression, reported alcohol and substance use, and social support of Anesthesiologists. Anesth Analg 2017;125:2009-18.

8 Panagioti M, Geraghty K, Johnson J, et al. Association between physician burnout and patient safety, professionalism, and patient satisfaction: a systematic review and meta-analysis. JAMA Intern Med 2018;178:1317-30.

9 Azoulay E, De Waele J, Ferrer R, et al. Symptoms of burnout in intensive care unit specialists facing the COVID-19 outbreak. Ann Intensive Care 2020;10:1-8.

10 Dzeng E, Curtis JR. Understanding ethical climate, moral distress, and burnout: a novel tool and a conceptual framework. BMJ Qual Saf 2018;27:766-70.

11 Fourie C. Moral distress and moral conflict in clinical ethics. Bioethics 2015;29:91-7.

12 Fumis RRL, Junqueira Amarante GA, de Fátima Nascimento A, et al. Moral distress and its contribution to the development of burnout syndrome among critical care providers. Ann Intensive Care 2017;7:1-8.

13 Quenot JP, Rigaud JP, Prin S, et al. Suffering among carers working in critical care can be reduced by an intensive communication strategy on end-of-life practices. Intensive Care Med 2012;38:55-61.

14 Dryden-Palmer K, Moore G, McNeil C, et al. Moral distress of clinicians in Canadian pediatric and neonatal ICUs. Pediatr Crit Care Med 2020;21:314-23.

15 Van den Bulcke B, Metaxa V, Reyners AK, et al. Ethical climate and intention to leave among critical care clinicians: an observational study in 68 intensive care units across Europe and the United States. Intensive Care Med 2020;46:46-56.

16 Mealer M, Moss M. Moral distress in ICU nurses. Intensive Care Med 2016;42:1615-7.

17 Panagioti M, Panagopoulou E, Bower P, et al. Controlled interventions to reduce burnout in physicians: a systematic review and meta-analysis. JAMA Intern Med 2017;177:195-205.

18 Sexton JB, Adair KC. Forty-five good things: a prospective pilot study of the three good things well-being intervention in the USA for healthcare worker emotional exhaustion, depression, work-life balance and happiness. BMJ Open 2019;9:e022695.

19 van der Wal RAB, Bucx MJL, Hendriks JCM, et al. Psychological distress, burnout and personality traits in Dutch anaesthesiologists: a survey. Eur J Anaesthesiol 2016;33:179-86.

20 Zellars KL, Perrewé PL, Hochwarter WA. Burnout in health care: the role of the five factors of personality. Journal of Applied Psychology 2000;30:1570-98.

21 Schwartz SP, Adair KC, Bae J, et al. Work-life balance behaviours cluster in work settings and relate to burnout and safety culture: a cross-sectional survey analysis. BMJ Qual Saf 2019;28:142-50.

22 Neumann JL, Mau L-W, Virani S, et al. Burnout, moral distress, work-life balance, and career satisfaction among hematopoietic cell transplantation professionals. Biol Blood Marrow Transplant 2018;24:849-60.

23 Del Carmen MG, Herman J, Rao S, et al. Trends and factors associated with physician burnout at a Multispecialty 
academic faculty practice organization. JAMA Netw Open 2019;2:e190554.

24 Sexton JB, Sharek PJ, Thomas EJ, et al. Exposure to leadership WalkRounds in neonatal intensive care units is associated with a better patient safety culture and less caregiver burnout. BMJ Qual Saf 2014;23:814-22.

25 West CP, Dyrbye LN, Shanafelt TD. Physician burnout: contributors, consequences and solutions. J Intern Med 2018;283:516-29.

26 Törnroos M, Elovainio M, Hintsa T, et al. Personality traits and perceptions of organisational justice. Int J Psychol 2019;54:414-22.

27 Schaufeli W, Van Dierendonck D. Utrecht Burnout Scale, Manual [Dutch]. Utrecht: Swets \& Zeitlinger, 2000.

28 Hamric AB, Borchers CT, Epstein EG. Development and testing of an instrument to measure moral distress in healthcare professionals. AJOB Prim Res 2012;3:1-9.

29 Colville GA, Dawson D, Rabinthiran S, et al. A survey of moral distress in staff working in intensive care in the UK. $J$ Intensive Care Soc 2019;20:196-203.

30 Hahn E, Gottschling J, Spinath FM. Short measurements of personality - validity and reliability of the GSOEP big five inventory (BFI-S). J Res Pers 2012;46:355-9.

31 Demerouti E, Geurts SAE, Kompier M. Positive and negative work-home interaction: prevalence and correlates. Equal Opportunities Int 2004;23:6-35.

32 Geurts SAE, Taris TW, Kompier MAJ, et al. Work-home interaction from a work psychological perspective: development and validation of a new questionnaire, the swing. Work \& Stress 2005;19:319-39.

33 Rafferty AM, Philippou J, Fitzpatrick JM, et al. Development and testing of the 'Culture of Care Barometer' (CoCB) in healthcare organisations: a mixed methods study. BMJ Open 2017;7:e016677.

34 Haerkens MH, van Leeuwen W, Sexton JB, et al. Validation of the Dutch language version of the safety attitudes questionnaire (SAQ-NL). BMC Health Serv Res 2016;16:385.

35 Laurent E, Schonfeld IS, Bianchi R, et al. On the "bubble" of burnout's prevalence estimates. Intensive Care Med 2018;44:544-5.

36 Rotenstein LS, Torre M, Ramos MA, et al. Prevalence of burnout among physicians: a systematic review. JAMA 2018;320:1131-50.

37 van Mol MMC, Kompanje EJO, Benoit DD, et al. The prevalence of compassion fatigue and burnout among healthcare professionals in intensive care units: a systematic review. PLoS One 2015;10:e0136955.

38 Rubin M. Do $p$ Values Lose Their Meaning in Exploratory Analyses? It Depends How You Define the Familywise Error Rate. Review of General Psychology 2017;21:269-75.

39 Hayes AF, Rockwood NJ. Regression-based statistical mediation and moderation analysis in clinical research: observations, recommendations, and implementation. Behav Res Ther 2017;98:39-57.

40 Embriaco N, Azoulay E, Barrau K, et al. High level of burnout in intensivists: prevalence and associated factors. Am J Respir Crit Care Med 2007;175:686-92.

41 Poncet MC, Toullic P, Papazian L, et al. Burnout syndrome in critical care nursing staff. Am J Respir Crit Care Med 2007;175:698-704.

42 Merlani P, Verdon M, Businger A, et al. Burnout in ICU caregivers: a multicenter study of factors associated to centers. Am J Respir Crit Care Med 2011;184:1140-6.

43 Ricou B, Gigon F, Durand-Steiner E, et al. Initiative for burnout of ICU caregivers: feasibility and preliminary results of a psychological support. J Intensive Care Med 2020;35:562-9.

44 Verdon M, Merlani P, Perneger T, et al. Burnout in a surgical ICU team. Intensive Care Med 2008;34:152-6.

45 Haerkens MHTM, Kox M, Lemson J, et al. Crew resource management in the intensive care unit: a prospective 3-year cohort study. Acta Anaesthesiol Scand 2015;59:1319-29.

46 Omar AS, Elmaraghi S, Mahmoud MSA, et al. Impact of leadership on ICU clinicians' burnout. Intensive Care Med 2015;41:2016-7.

47 Cummings G, Hayduk L, Estabrooks C. Mitigating the impact of hospital restructuring on nurses: the responsibility of emotionally intelligent leadership. Nurs Res 2005;54:2-12.

48 Maslach C, Schaufeli WB, Leiter MP. Job burnout. Annu Rev Psychol 2001;52:397-422.

49 Li CY, Sung FC. A review of the healthy worker effect in occupational epidemiology. Occup Med 1999;49:225-9.

50 Pedersen AF, Sørensen JK, Bruun NH, et al. Risky alcohol use in Danish physicians: associated with alexithymia and burnout? Drug Alcohol Depend 2016;160:119-26.

51 Vandenbroeck S, Van Gerven E, De Witte H, et al. Burnout in Belgian physicians and nurses. Occup Med 2017;67:546-54.

52 Pantenburg B, Luppa M, König H-H, et al. Burnout among young physicians and its association with physicians' wishes to leave: results of a survey in Saxony, Germany. J Occup Med Toxicol 2016;11.

53 Meynaar IA, Ottens T, Zegers M, et al. Burnout, resilience and work engagement among Dutch intensivists in the aftermath of the COVID-19 crisis: a nationwide survey. J Crit Care 2021;62:1-5.

54 Twellaar M, Winants Y, Houkes I. How healthy are Dutch general practitioners? self-reported (mental) health among Dutch general practitioners. Eur J Gen Pract 2008;14:4-9.

55 Dykema J, Jones NR, Piché T, et al. Surveying clinicians by web: current issues in design and administration. Eval Health Prof 2013;36:352-81.

56 Haan MM, van Gurp JLP, Naber SM, et al. Impact of moral case deliberation in healthcare settings: a literature review. BMC Med Ethics 2018;19:1-15. 